HELMINTHOLOGIA, 55, 1: 12 - 20, 2018

\title{
High IL-1a production was induced in the WBN/Kob-Lepr fa type 2 diabetes mellitus rat model and inhibited by Syphacia muris infection
}

\author{
M. OKAMOTO ${ }^{1, \S, *}$, R. ITO ${ }^{1, \S}$, K. TAIRA $^{2}$, T. IKEDA ${ }^{1}$ \\ 1 Laboratory of Veterinary Immunology, Department of Veterinary Medicine, School of Veterinary Medicine, Azabu University, \\ 1-17-71 Fuchinobe, Chuo-ku, Sagamihara, Kanagawa 252-5201, Japan, E-mail: m-okamoto@azabu-u.ac.jp; \\ 2Laboratory of Parasitology, Department of Veterinary Medicine, School of Veterinary Medicine, Azabu University, \\ 1-17-71 Fuchinobe, Chuo-ku, Sagamihara, Kanagawa 252-5201, Japan
}

Article info

Received March 3, 2017

Accepted September 5, 2017

\begin{abstract}
Summary
The novel WBN/Kob-Lepra (fa/fa) congenic rat strain is considered a useful rat model of type 2 diabetes mellitus (T2DM). Accumulating findings suggest that low-grade inflammation is a causative factor in T2DM and that circulating levels of inflammatory cytokines are associated with insulin resistance. However, inflammatory cytokine profiles and their correlations with T2DM development/ progression in fa/fa rats have not been studied. In this study, we found that the fa/fa rats had considerably high plasma levels of interleukin (IL)-1a. Abundant cecal IL-1a mRNA expression and cecal inflammation with infiltrating IL-1a-producing macrophages was observed in fa/fa rats. Bone marrow derived macrophages from fa/fa rats expressed high levels of IL-1 a upon lipopolysaccharide stimulation. Furthermore, Syphacia muris infection, which delays the onset of T2DM, reduced both plasma and cecal IL-1a levels in fa/fa rats. These results suggest that macrophage infiltration and IL-1a secretion comprise an important part of T2DM development and that $S$. muris infection inhibits pro-inflammatory cytokine expression in fa/fa rats.
\end{abstract}

Keywords: IL-1a; macrophage; Syphacia muris; type 2 diabetes mellitus; WBN/Kob-Lepra

\section{Introduction}

Type 2 diabetes mellitus (T2DM) is a major international health problem with an increasing global prevalence (Farag et al., 2011, Zimmet et al., 2001). Approximately $95 \%$ of patients with diabetes mellitus suffer from T2DM, which is characterized by insulin resistance and pancreatic $\beta$-cell dysfunction. Recent studies have demonstrated that chronic low-grade inflammation with elevated levels of pro-inflammatory cytokines contributes to the onset and progression of T2DM (Donath et al., 2011). For example, interleukin (IL)-1 $\beta$, a representative pro-inflammatory cytokine, is produced via the Nod-like receptor (NLR) family pyrin domain containing 3 (NLRP3) inflammasome pathway following activation by free fatty acids (Wen et al., 2011). IL-1 $\beta$ leads macrophage recruitment and islet $\beta$-cell death by inducing upregulation of the pro-apoptotic receptor Fas (Maedler et al., 2002). Anti-inflammatory approaches that target specific pro-inflammatory cytokine pathways are expected to be efficacious in the treatment or prevention of T2DM.

Animal models of T2DM are needed to elucidate pathogenesis and conduct pharmacological studies of new candidates for therapeutic or preventive options. The WBN/Kob-Lepr ${ }^{f a}(f a / f a)$ rat is a new congenic strain established by introducing the leptin receptor fatty gene $\left(L p r^{f a}\right)$, a spontaneous mutation derived from Zucker-fatty rats that develop obesity and hyperinsulinemia consequent to leptin receptor dysfunction (Zucker, 1965; Chua et al., 1996), into the parental WBN/Kob rat genome (Akimoto et al., 2008). Previous reports revealed that fa/fa rats develop hyperglycemia at 9 weeks of age, evidenced by impaired glucose tolerance, insulin resistance, and dyslipidemia in a manner similar to human T2DM8 (Akimoto et al., 2008; Akimoto et al., 2012; Kaji et al., 2012; Okuno

\footnotetext{
* - corresponding author

$\S$ - These authors contributed equally to this work.
} 
et al., 2013; Nagakubo et al., 2014), thus suggesting that the fa/fa rat is useful as a new model of T2DM.

In a previous study, we demonstrated that infection with Syphacia (S.) muris, a nematode that targets rats, delayed the onset of hyperglycemia in fa/fa rats (Taira et al., 2015). Furthermore, a global transcriptomic next-generation sequencing analysis of $S$. muris suggested that some genes expressed in $S$. muris might modify the host immune response; these genes include a gene involved in the regulation of macrophage activation via the Fc receptor signaling pathway (Okamoto et al., 2015). Those studies suggested that $S$. muris infection might regulate the local production of cytokines and chemokines that promote the development of T2DM. However, the inflammatory cytokine profiles and their contributions to the development and progression of T2DM have not been investigated in fa/fa rats. The aim of present study, therefore, was to evaluate cytokine/chemokine profiles in fa/fa rats and compare them with profiles of control Wistar rats or S. muris-infected fa/fa rats. In addition, cells, organs or tissues, and/or tissues that produce inflammatory cytokines in fa/fa rats were also investigated.

\section{Materials and Methods}

\section{Animals}

Male WBN/Kob-Lepra (fa/fa) and Wistar rats (control) were obtained from SLC Inc. (Shizuoka, Japan). All rats were maintained under conventional conditions with a $12 \mathrm{~h}$ on/off light cycle, commercial diet, and water ad libitum. All animal studies were approved by the Azabu University Animal Research Ethics Committee (approval No. 150303-3). All animal procedures were performed under the experimental animal guidelines of Azabu University. Blood glucose levels of fa/fa rats were measured weekly.

\section{Parasites and infection}

S. muris infection was performed as described previously (Taira et al., 2015). Briefly, S. muris eggs were harvested from the uteruses of female worms obtained from the colons and rectums of experimentally infected male Wistar rats (Stahl, 1961). At 5 weeks of age, fa/fa rats were inoculated once via stomach tubes with either approximately 100 eggs $/ \mathrm{ml} /$ rat or $1 \mathrm{ml} /$ rat of distilled water (for uninfected group). S. muris infection was confirmed using cellophane tape to detect pinworm eggs in the perianal regions of rats.

\section{Blood sampling}

Blood samples were collected from the tail vein, and plasma samples were obtained by centrifuging heparinized blood. In some experiments we used Wistar rats' plasma purchased from SLC Inc. as a "control."

\section{Cytokine and chemokine array}

To measure plasma cytokine and chemokine levels, pooled plasma from 9 - 10-week-old fa/fa rats, $S$. muris-infected fa/fa rats, and control Wistar rats plasma (SLC Inc.) were used. Cytokine profiles were analyzed using the Proteome Profiler ${ }^{\mathrm{TM}}$ Rat Cytokine Array Panel A (R\&D Systems, Wiesbaden, Germany), which can identify 29 cytokines (Fig. 1A). All experiments were performed according to the manufacturer's instructions. Spots were visualized using Immobilon Western (Merck Millipore, Massachusetts, USA) and captured with an ImageQuant LAS-4000 (GE Healthcare, Buckinghamshire, UK). ImageQuant TL software (GE Healthcare) was used for the data analysis. Signals were normalized using internal controls included on the array.

\section{Enzyme-linked immunosorbent assay (ELISA)}

IL-1a levels in plasma or bone marrow-derived macrophage (BMDM) culture supernatant were quantified using a Rat IL-1a Platinum ELISA (eBioscience, San Diego, CA, USA) according to the manufacturer's instructions.

\section{Quantitative real-time PCR (RT-qPCR)}

RT-qPCR of IL-1a was performed as described previously (Okamoto et al., 2009), using RNA from ceca isolated from 10-week-old fa/fa and S. muris-infected fa/fa rats and Wistar rats. Total RNA was extracted from each cecum using an RNeasy Mini kit (QIAGEN, Hilden, Germany). Single-stranded cDNA was synthesized from total RNA using the RT2 First Strand Kit (QIAGEN). IL-1a mRNA expression was determined by RT-qPCR with the RT2 SYBR Premix and primer sets for IL-1a (ref.seq. NM_017019.1) and $\beta$-actin (ref.seq. NM_031144.2) or 18s RNA (ref.seq. X01117.1); these reagents were purchased from QIAGEN and used according to the manufacturer's instructions. Relative target gene quantification was calculated according to the comparative CT method.

\section{Histologic analysis}

Cecal tissues were isolated from 12-16-week-old fa/fa rats and Wistar rats. Tissues were fixed in a $10 \%$ neutral buffered formalin solution and embedded in paraffin blocks or, for frozen sections, in OCT compound (Sakura FineTek, Tokyo, Japan) after snap-freezing in liquid nitrogen as described previously (Nagakubo et al.,

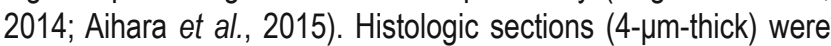
stained with hematoxylin and eosin (HE).

\section{Immunofluorescence analysis}

To detect IL-1a expression in the cecum, frozen cecal sections were incubated with a rabbit anti-IL-1 antibody (Santa Cruz Biotechnology, Dallas, TX, USA). Alexa Flour 488-conjugated goat anti-rabbit IgG (Invitrogen, Carlsbad, CA, USA) was used for secondary detection. To detect macrophages in the cecum, frozen sections were incubated with mouse anti-CD68 (Serotec, Oxford, UK). Alexa Flour 568-conjugated goat anti-mouse IgG (Invitrogen) was used for secondary detection. Sections were mounted in VECTASHIELD Mounting Medium with DAPI (Vector Laboratories, Burlingame, CA, USA). Stained sections were examined and imaged using a fluorescence microscope (FSX100; Olympus, Tokyo, Japan). 
A

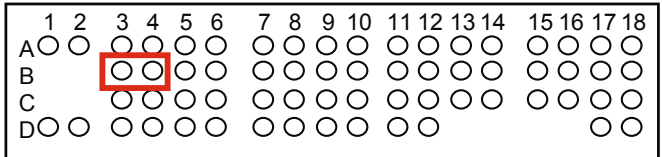

\begin{tabular}{|c|c|c|c|}
\hline coordinate & target & coordinate & target \\
\hline $\mathrm{A} 1, \mathrm{~A} 2$ & control & $\mathrm{C} 3, \mathrm{C} 4$ & IL-13 \\
\hline $\mathrm{A} 3, \mathrm{~A} 4$ & CINC-1 & $\mathrm{C} 5, \mathrm{C} 6$ & IL-17 \\
\hline $\mathrm{A} 5, \mathrm{~A} 6$ & CINC- $2 \alpha / \beta$ & $\mathrm{C} 7, \mathrm{C} 8$ & CXCL10 \\
\hline $\mathrm{A7}, \mathrm{A} 8$ & CINC-3 & $\mathrm{C} 9, \mathrm{C} 10$ & LIX \\
\hline $\mathrm{A} 9, \mathrm{~A} 10$ & CNTF & $\mathrm{C} 11, \mathrm{C} 12$ & L-Selectin \\
\hline $\mathrm{A} 11, \mathrm{~A} 12$ & CX3CL1 & C13, C14 & CXCL9 \\
\hline $\mathrm{A} 13, \mathrm{~A} 14$ & GM-CSF & C15, C16 & CCL3 \\
\hline $\mathrm{A} 15, \mathrm{~A} 16$ & sICAM-1 & C17, C18 & CCL20 \\
\hline A17, A18 & IFN- $\gamma$ & $\mathrm{D} 1, \mathrm{D} 2$ & control \\
\hline B3, B4 & |L-1 $\alpha$ & D3, D4 & CCL5 \\
\hline $\mathrm{B} 5, \mathrm{~B} 6$ & IL-1 $\beta$ & D5, D6 & CXCL7 \\
\hline $\mathrm{B} 7, \mathrm{~B} 8$ & IL-1ra & $\mathrm{D} 7, \mathrm{D} 8$ & TIMP-1 \\
\hline B9, B10 & IL-2 & $\mathrm{D9}, \mathrm{D} 10$ & TNF- $\alpha$ \\
\hline B11, B12 & IL-3 & D11, D12 & VEGF \\
\hline B13, B14 & IL-4 & \multirow{2}{*}{ D17, D18 } & \multirow{2}{*}{ negative contro } \\
\hline B15, B16 & IL-6 & & \\
\hline B17, B18 & IL-10 & & \\
\hline
\end{tabular}

B
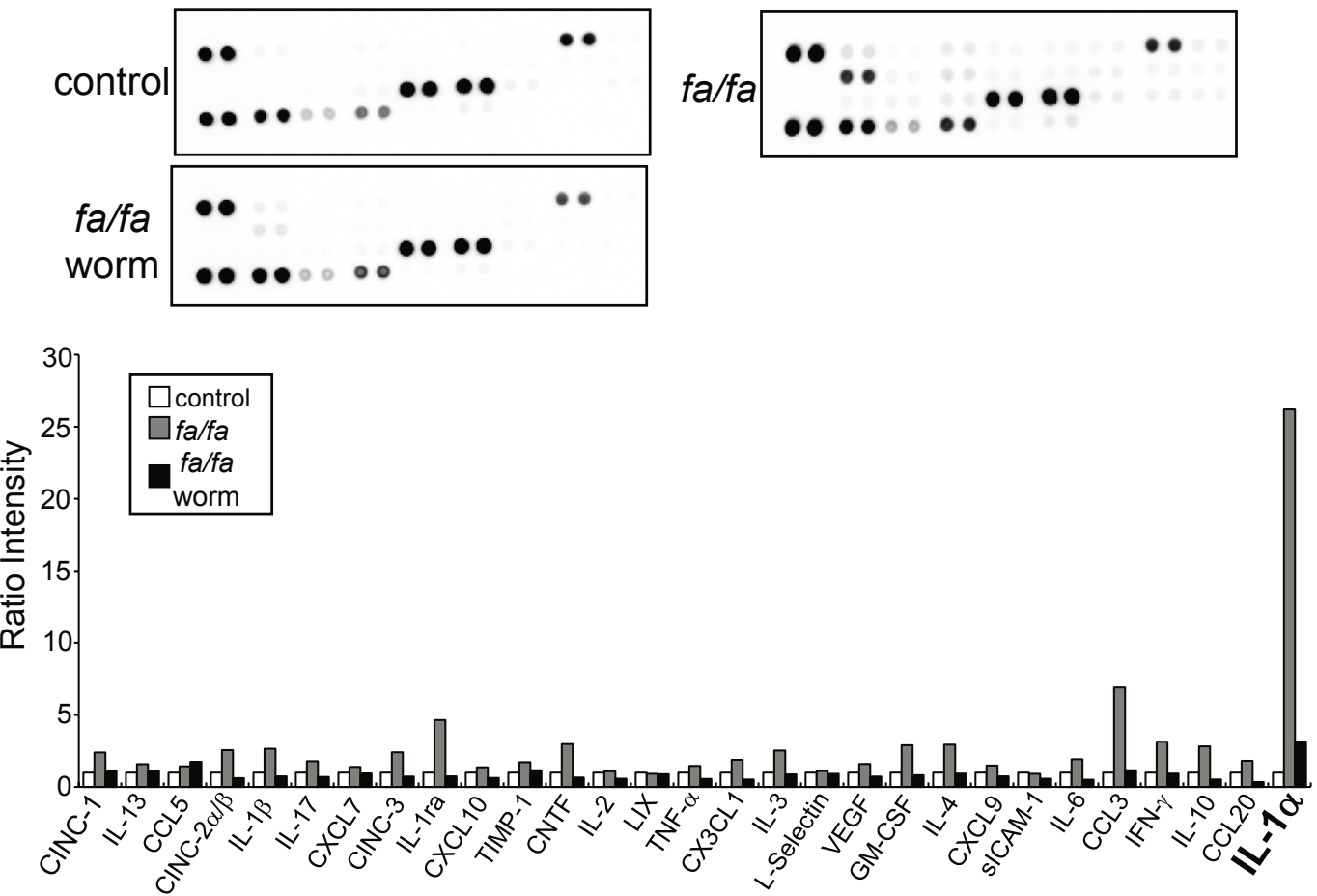

Fig. 1. Profile of circulating cytokines and chemokines in rats according to an array panel

(A) Rat cytokine/chemokine array panel coordinates. The IL-1 $\alpha$ coordinate is indicated by a box with red line.

(B) Plasma cytokines and chemokines from Wistar rats (control), fa/fa rats (fa/fa), and Syphacia muris-infected fa/fa rats (fa/fa worm) were measured using a rat cytokine/chemokine array panel. The signal intensity at each spot was normalized using internal controls included on the array, and relative levels of each cytokine/chemokine are presented as a graph. 


\section{Macrophage differentiation and stimulation}

BMDMs were generated from bone marrow cells collected from the femurs of 8-week- old fa/fa rats and Wistar rats, followed by in vitro differentiation in high-glucose Dulbecco's modified Eagle's medium (DMEM; Wako Pure Chemical Industries, Osaka, Japan) supplemented with $10 \%$ heat-inactivated fetal bovine serum, 100U/ $\mathrm{ml}$ penicillin/streptomycin, $2 \mathrm{mM} \mathrm{L-glutamine,} \mathrm{and} 10 \mathrm{ng} / \mathrm{ml}$ recombinant mouse macrophage colony-stimulating factor (M-CSF; Peprotech, Rocky Hill, NJ, USA). Cells were maintained in $5 \% \mathrm{CO} 2$ at $37^{\circ} \mathrm{C}$. On days $3-4$, BMDMs were counted and seeded into 24-well plates $\left(5 \times 10^{5}\right.$ cells/well). Cells were incubated overnight and then stimulated with $1 \mu \mathrm{g} / \mathrm{ml}$ of bacterial lipopolysaccharide (LPS, E.Coli O55:B5; Sigma-Aldrich, St. Louis, MO, USA). Cells and supernatants were harvested 24 hours after LPS stimulation for gene expression studies. IL-1a RT-qPCR and ELISA analyses were carried out as described above.

\section{Statistical analysis}

Data are presented as means \pm standard errors (SEs). The analysis involved a one-way analysis of variance (ANOVA) followed by Dunnett's multiple comparison test. Student's t test for paired observations was used for the statistical comparison of BMDC IL1a expression. Results were considered statistically significant at a $p$ value $<0.05$.

\section{Results}

Array panel comparison of inflammatory cytokines and chemokines

We first valuated plasma cytokine/chemokine profiles of WBN/ Kob-Lepr ${ }^{f a}(\mathrm{fa} / \mathrm{fa})$ rats that developed diabetes, characterized by high plasma glucose levels at 9 weeks of age, using the cytokine/ chemokine array panel (Fig.1A). As shown in Fig. 1B, the array analysis revealed very low levels of cytokines and chemokines

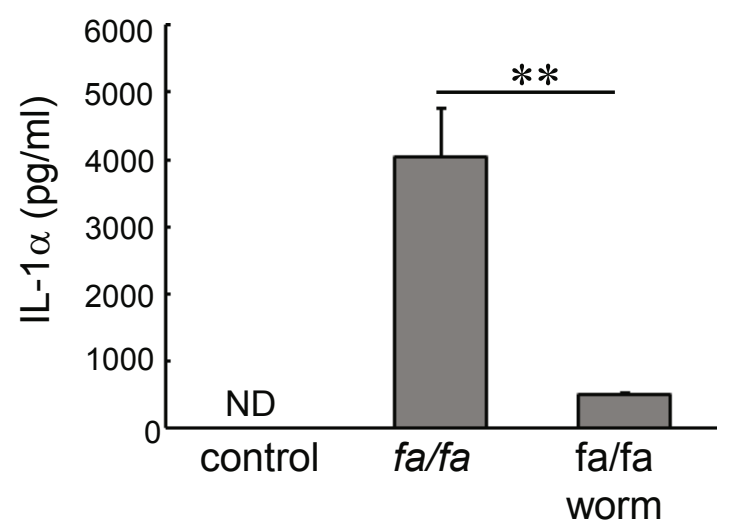

Fig. 2. Plasma IL-1a protein levels in control, fa/fa and Syphacia (S.) murisinfected fa/fa rats. Plasma IL-1 $\alpha$ levels in Wistar rats (control), fa/fa rats (fa/fa), and $S$. muris-infected fa/fa rats (fa/fa worm) were measured by enzyme-linked immunosorbent assay. Data are presented as means \pm standard errors $(n=4)$. $* *: p<0.01 . \mathrm{ND}=$ not detected. in the plasma of control rats. In contrast, the plasma levels of most cytokines and chemokines were at least 2-fold higher in fa/fa rats than in control rats, although the signal at each spot was relatively weak (Fig. 1B). Among these elevated cytokines, we detected an extremely high level of IL-1a in plasma from fa/fa rats (Fig. $1 B$ ). The levels of $I L-1$ a was higher than that of $I L-1 \beta$, another $I L-1$ family cytokine that has been reported to associate with chronic inflammation in obesity (Fig. 1B).

fa/fa rats exhibit elevated plasma levels of IL-1 $\alpha$

We next examined the amount of IL-1a in plasma from control and fa/fa rats by ELISA. IL-1a was not detected in plasma from control

\section{A}

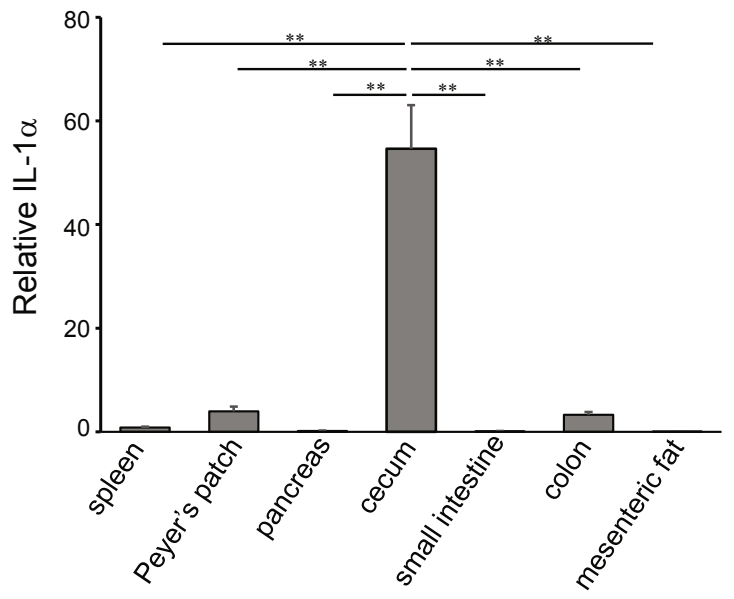

B

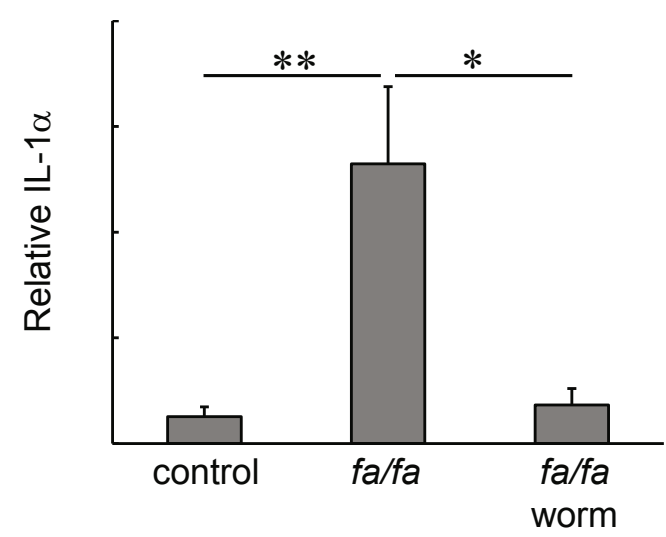

Fig. 3. Relative IL-1a mRNA levels in the ceca of control, fa/fa and Syphacia (S.) muris-infected fa/fa rats. (A) IL-1 $\alpha$ mRNA expression levels among organs and tissues (spleen, Peyer's patch, pancreas, cecum, intestine, colon, mesenteric fat) were measured by real-time quantitative PCR. (B) IL-1 $\alpha$ mRNA expression levels in the ceca of Wistar rats (control), fa/fa rats (fa/fa), and S. muris-infected fa/fa rats (fa/fa worm) were measured by real-time quantitative PCR.

Data are expressed as means \pm standard errors $(n=4) . *$ : $p<0.05, * *: p<0.01$. 
A

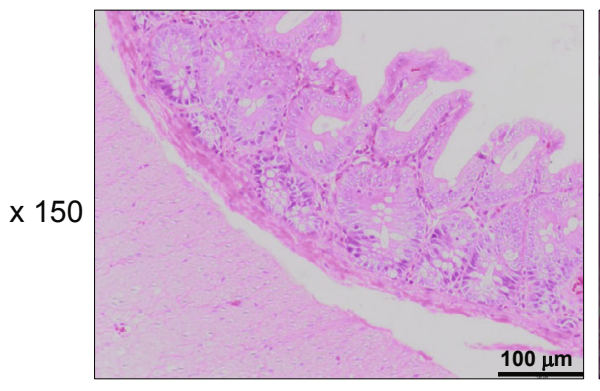

control

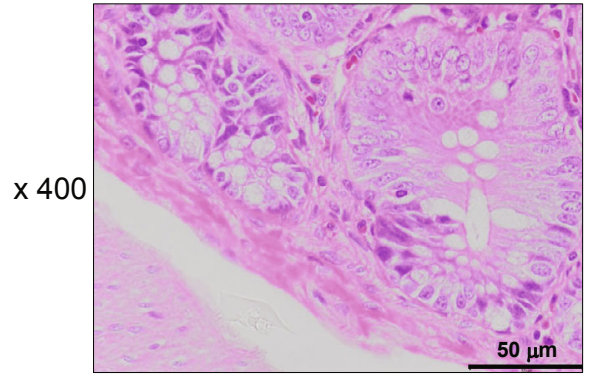

B

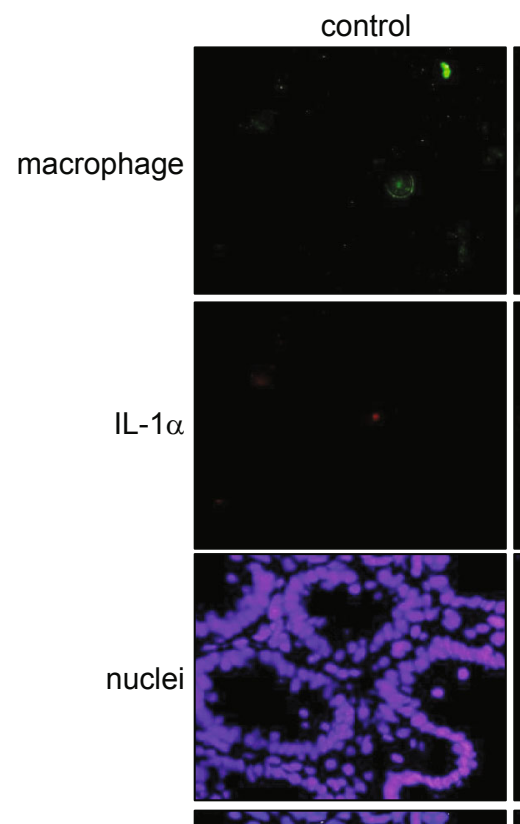

merge

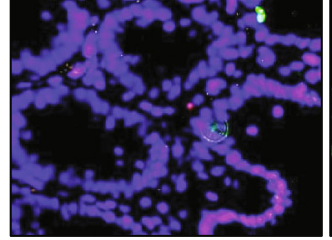

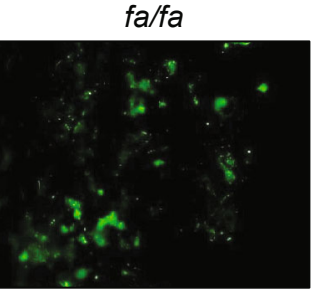

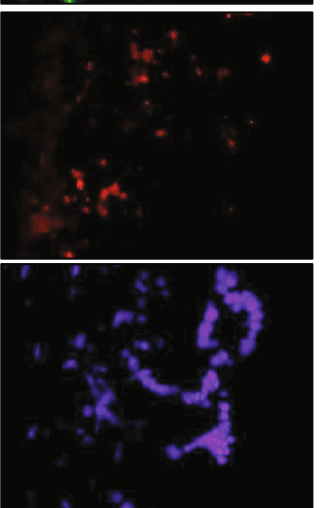

$\mathrm{fa} / \mathrm{fa}$
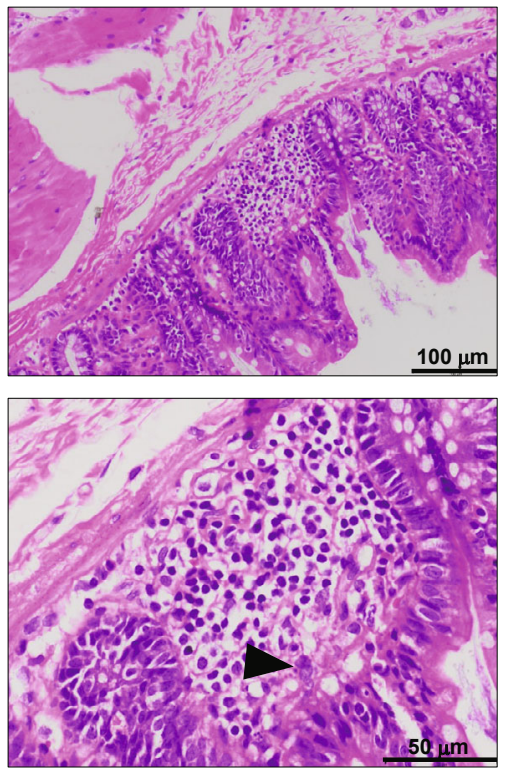

$\mathrm{fa} / \mathrm{fa}$

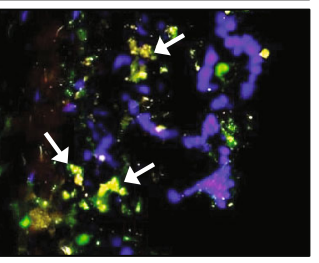

Fig. 4. Cecal inflammation with IL-1a producing-macrophage infiltration observed in fa/fa rats.

(A) Representative hematoxylin and eosin-stained cecal sections from control and fa/fa rats. Low-powered ( $x 150$, top) and high-powered ( $x 400$, bottom) images of ceca of Wistar rats (control) and fa/fa rats (fa/fa). Mucosal infiltrates of lymphoid cells and macrophages (arrowhead) are visible in the ceca of fa/fa rats. (B) Representative immunohistochemical images of F4/80 and IL-1 $\alpha$. Cecal sections of Wistar rats (control) or fa/fa rats (fa/fa) were stained with antibodies against macrophage marker F4/80 (green) and IL-1 $\alpha$ (red). Nuclei were stained with DAPI (blue). Images were captured at 150x magnification. Macrophages expressing IL-1 $\alpha$ (arrow) are visible in the ceca of fa/fa rats. 
rats (Fig. 2). On the other hand, the amount of plasma IL-1a in fa/fa rats was extremely elevated in comparison with control rats (Fig. 2); in other words, these results were similar to those obtained from the cytokine/chemokine array panel (Fig. 1B). These findings, taken together with the results in Fig. $1 \mathrm{~B}$, demonstrate that the pro-inflammatory cytokine IL-1a is the main cytokine produced in fa/fa rats that have developed diabetes.

\section{Ceca from fa/fa rats express high levels of IL-1 a mRNA}

We used RT-qPCR to investigate IL-1a expression in various organs of fa/fa rats in an attempt to identify the sources of the high levels of circulating IL-1a. Total RNA was extracted from various organs or tissues (including spleen, Peyer's patch, pancreas, cecum, intestine, colon, mesenteric fat) with suspected involvement in the production of inflammatory cytokines associated with

\section{A}

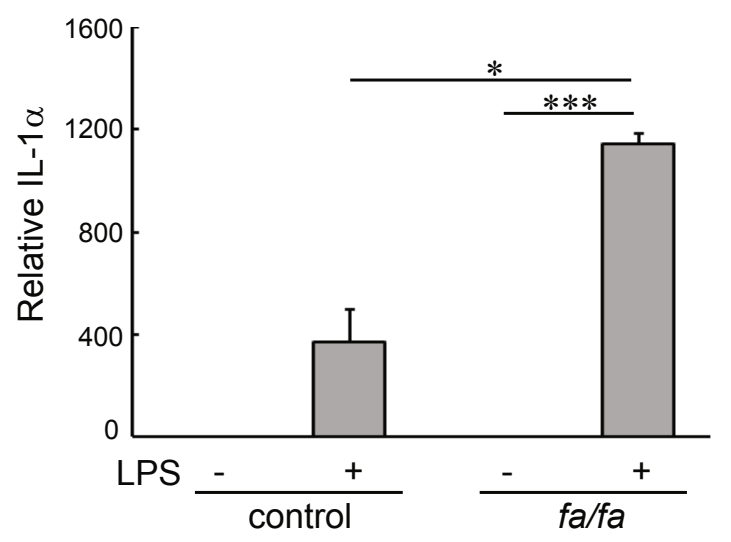

B

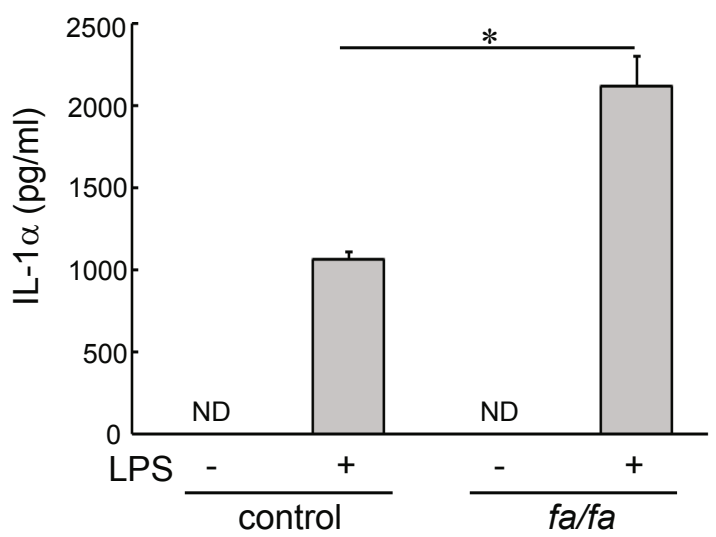

Fig. 5. Levels of IL-1a produced by bone marrow-derived macrophages (BMDMs) from control and fa/fa rats. BMDMs from Wistar rats (control) or fa/fa rats (fa/fa) were unstimulated or stimulated with LPS $(1 \mu \mathrm{g} / \mathrm{ml})$. (A) IL-1a mRNA expression levels were determined by real-time quantitative PCR. (B) IL-1a protein levels in supernatants were measured by enzyme-linked immunosorbent assay. Data are expressed as means \pm standard errors $(n=3)$. $*: p<0.05, * *: p<0.01, * * *: p<0.001$ the onset of T2DM. Among the organs and tissues analyzed in $\mathrm{fa} /$ fa rats, the highest levels of IL-1a were found in the cecum (Fig. $3 A)$. As shown in Fig. 3B, the IL-1a mRNA expression levels in the ceca of control rats were very low, whereas those of fa/fa rats were significantly increased. IL-1a expression was not detected in the spleen or pancreas in fa/fa rats (Fig. 3A). These results suggest that the cecum is one of the main sources of the high levels of plasma IL-1a observed in fa/fa rats.

fa/fa rats exhibit cecal inflammation with macrophage infiltration IL-1a has recently been reported to associate with sterile inflammation (Freigang et al., 2013). Because high levels of IL-1a were expressed in the ceca of fa/fa rats with diabetes, a histological analysis of the ceca from fa/fa rats was conducted to examine the presence of inflammation. These histological findings are shown in Fig. 4A. Pathological cecal changes were not observed in control rats. In contrast, the ceca of fa/fa rats exhibited inflammatory cell infiltration into the lamina propria (Fig. 4A). Although focal inflammatory infiltrates in the cecal mucosa were mainly composed of lymphocytes, macrophage infiltration was also observed (Fig. 4A, arrowhead). We further subjected cecal sections from control or $\mathrm{fa} / \mathrm{fa}$ rats with diabetes to an immunohistochemical analysis with antibodies against the macrophage marker F4/80 and against the C-terminal of IL-1a. As shown in Fig. 4B, fa/fa rats contained increased numbers of F4/80-positive macrophages in the cecal mucosa, compared with control rats. Furthermore, IL-1a protein-producing macrophages were detected in cecal sections of fa/fa rats, but not of control rats (arrows in Fig. 4B). These results suggest that macrophage infiltration and consequent enhanced IL-1a production might induce cecal inflammation in fa/fa rats.

Increased IL-1a production from fa/fa BMDMs upon LPS stimulation

Thus far, our results suggested that pro-inflammatory responses of macrophages are enhanced in fa/fa rats relative to control rats. To evaluate this further, we stimulated BMDMs from fa/fa or control rats with bacterial lipopolysaccharide (LPS), which induces proinflammatory cytokine production through toll-like receptor (TLR) 4 activation (Jin et al., 2008; Kawai et al., 2011). We observed no differences in macrophage differentiation rates, which were determined using a macrophage-specific antibody against F4/80 (Santa Cruz Biotechnology), between fa/fa or control rats. Very low levels of IL-1a mRNA were detected in the absence of stimulation, and IL1a expression was induced by LPS stimulation in BMDCs from both genotypes (Fig. 6A). However, the IL-1a mRNA expression levels in $\mathrm{fa} / \mathrm{fa} \mathrm{BMDCs}$ upon stimulation were significantly higher than those in control BMDCs (Fig. 6A). We also measured the amount of IL-1a protein in culture supernatants; as shown in Fig. 6B, culture supernatants from LPS-stimulated fa/fa BMDCs contained much higher amounts of IL-1a than did those from stimulated control BMDCs. These findings indicate that macrophages derived from fa/fa rats exhibit enhanced responsiveness to pro-inflammatory stimuli. 
fa/fa rats infected with $S$. muris decreases plasma cytokine/ chemokine levels and especially prominent elevated level of IL-1a in plasma and cecum

In a previous study, we demonstrated that infection with $S$. muris delayed the onset of T2DM in fa/fa rats, and suggested that this nematode infection might regulate the local production of cytokines/chemokines production that promote T2DM development (Taira et al., 2015). To examine the effect of $S$. muris infection on cytokine/chemokine production in fa/fa rats, we first investigated changes in plasma cytokine/chemokine levels in fa/fa and S. muris-infected fa/fa rats. An array analysis of circulating cytokines/ chemokines revealed that when compared with uninfected $\mathrm{fa} /$ fa rats, the plasma levels of most cytokines, including IL-1 $\beta$ and chemokines were decreased in $S$. muris-infected fa/fa rats to the levels similar to those detected in control rats (Fig. 1B). Furthermore, we found that the levels of $\mathrm{IL}-1 \mathrm{a}$ which were extremely high in fa/fa rats, were also dramatically reduced in S. muris-infected fa/fa rats (Fig. 1B). ELISA analysis also demonstrated a remarkable decrease in the plasma IL-1a concentration in $\mathrm{fa} / \mathrm{fa}$ rats infected with S. muris, compared with uninfected rats (Fig. 2). These results indicate that $S$. muris infection decreases the levels of circulating IL-1a in fa/fa rats. We further analyzed IL-1a mRNA levels by RT-qPCR to determine whether $S$. muris infection would affect IL-1a expression in the ceca of fa/fa rats. As shown in Fig. 3 , the relative expression level of IL-1a mRNA in the ceca of $S$. muris-infected fa/fa rats was significantly reduced when compared with that observed in uninfected rats, and was similar to the level detected in control rats. These findings suggest that $S$. muris infection inhibits the expression of cecal IL-1a in fa/fa rats with diabetes; in addition, this inhibition might delay the onset of T2DM by reducing cecal inflammation.

\section{Discussion}

Currently, chronic inflammation is well accepted as a critical factor in the onset and progression of T2DM (Donath et al., 2011). Chronic inflammation is characterized by an increase in circulating inflammatory cytokines (Rodriguez-Hernandez et al., 2013). In this study, we observed increased plasma levels of cytokines/chemokines in fa/fa rats with diabetes relative to those in age-matched control rats (Fig.1B). Likely, this increase in circulating cytokines results from pancreatic inflammation, as this strain naturally exhibits pancreatitis at $7-9$ weeks of age (Akimoto et al., 2012). We detected high plasma levels of IL-1a rather than IL-1 $\beta$ which is known to associate with T2DM (Fig.1B and Fig. 2). Although the circulating $\mathrm{IL}-1 \beta$ level was lower than the IL-1a level in fa/fa rats, it remained almost 2.6-fold higher than the plasma IL-1 $\beta$ level in control rats (Fig. 1B). This result suggests that IL-1 $\beta$ might also be involved in the onset of diabetes in fa/fa rats.

Aberrant IL-1 a signaling is involved in a wide range of inflammatory diseases, including arthritis, atherosclerosis, and diabetes (Kamari et al., 2011; Kamari et al., 2007; Banerjee et al., 2012; Rider et al.,
2013). However, when compared with IL-1 $\beta$, little is known about the contribution of IL-1a to the development of T2DM. Recently, Freigang et al., reported that IL-1a was selectively produced and secreted from macrophages upon stimulation via fatty acids such as oleic acid, leading to atherogenesis via vascular inflammation (Freigang et al., 2013; Spears et al., 2013). In our present study, cecal IL-1a expression was significantly higher in fa/fa rats with diabetes than in control rats (Fig. 3). Our data demonstrated elevated macrophage infiltration into the ceca of fa/fa rats, compared with control rats; in addition, the cecal macrophages in $\mathrm{fa} /$ fa rats expressed high levels of IL-1a (Fig. 4). Furthermore, IL-1a production in BMDMs derived from fa/fa rats in response to innate immune stimulation was significantly higher than in control BMDMs (Fig. 5). Recently, Jourdan et al., reported that endocannabinoid-activated infiltrating macrophages led to islet $\beta$-cell loss in a rat model of T2DM (Jourdan et al., 2013), suggesting that infiltrating macrophages and the consequent inflammatory cytokines play an important role in the onset of T2DM. Although the molecular mechanisms that control the increased production of IL-1a from macrophages of $\mathrm{fa} / \mathrm{fa}$ rats remains unclear, previous report noted that activation of the absent in melanoma 2 (AIM2) inflammasome led to the release of higher levels of IL-1a but not IL-1 $\beta$ via calpain activation (Sorrentino et al., 2015). Likewise, our results suggest that in fa/fa rats, macrophages are activated by stimuli such as free fatty acids from adipocytes or islet amyloid polypeptides, thus inducing IL-1a expression and secretion along with migration to the cecum.

We previously demonstrated that $S$. muris infection decreases plasma glucose level and delays the onset of T2DM in male fa/ fa rats (Taira et al., 2015). In the present study, S. muris-infected fa/fa rats exhibited decreased levels of circulating cytokines, including IL-1 $\alpha$ and IL-1 $\beta$ and chemokines (Fig. 1B and Fig. 2). Furthermore, S. muris-infected fa/fa rats exhibited reduced cecal IL-1a expression (Fig. 3B). These results suggest that $S$. muris could regulate the production of IL-1a in the cecum, the site of parasitization for this nematode. Our previous study revealed that S. muris expresses mRNAs for Fc receptor signaling proteins that might control macrophage activation (Okamoto et al., 2015). This report and our present study suggest that cecal macrophages are a candidate target for $S$. muris-mediated suppression of inflammatory reactions. We have not yet examined whether $S$. muris directly interacts with and regulates macrophage activation. In addition, it remains unclear whether a correlation exists between the cecal expression of IL-1a and pancreatitis. Future studies intended to identify the involvement of IL-1a or IL-1a-producing macrophages in pancreatitis and the onset of T2DM and to elucidate the molecular mechanism underlying the $S$. muris-mediated inhibition of inflammatory cytokine production will be indispensable.

\section{Conflict of interest}

None of the authors have any conflict of interests. 


\section{Acknowledgements}

We thank Dr. Fumitoshi Asai for his insightful discussions and experimental supports. We also thank Drs. Naoyuki Aihara, Noriaki Okamoto, Junichi Kamiie, Kinji Shirota, Mariko Shirota and Go Sugahara for their thoughtful comments and help; Mrs. Ayaka Watanabe and Mr. Yūki Ishikawa for technical supports. This study was partially supported by a project grant (Young Scientist Research Training Award) to MO funded by the Azabu University Research Services Division.

\section{References}

Aihara, N., Kamile, J., Yamada, M., Shirota, K. (2015): The development of mixed cryoglobulinemia in Capillaria hepatica-infected mice is associated with the capillaria antigen-induced selective proliferation of splenic B-1a cells in response to interleukin-5 stimulation. Am. J. Pathol., 185: 172 - 184. DOI: 10.1016/j.ajpath.2014.09.017

Akimoto, T., Nakama, K., Katsuta, Y., Zhang, X. J., Ohsuga, M., ISHIZAKI, M., SaWAI, N., OzaWA, H. (2008): Characterization of a novel congenic strain of diabetic fatty (WBN/Kob-Lepr ${ }^{\text {fa }}$ ) rat. Biochem. Biophys. Res. Commun., 366: 556 - 562. DOI: 10.1016/j. bbrc.2007.12.003

Aкimoto, T., Terada, M., Shimizu, A. (2012): Progression of pancreatitis prior to diabetes onset in WBN/Kob-Lepr(fa) rats. J. Vet. Med. Sci., 74: 65 - 70. DOI: 10.1292/jvms.11-0168

BanerJee, M., Saxena, M. (2012): Interleukin-1 (IL-1) family of cytokines: role in type 2 diabetes. Clin. Chim. Acta, 413: 1163-1170. DOI: 10.1016/j.cca.2012.03.021

Chua, S. C., JR., White, D. W., Wu-Peng, X. S., Liu, S. M., Okada, N., Kershaw, E. E., Chung, W. K., Power-Kehoe, L., Chua, M., TARTAglia, L. A., LeiBEL, RL. (1996): Phenotype of fatty due to Gln269Pro mutation in the leptin receptor (Lepr). Diabetes, 45: 1141 - 1143. DOI: 10.2337/diab.45.8.1141

Donath, M. Y., Shoelson, S. E. (2011): Type 2 diabetes as an inflammatory disease. Nat. Rev. Immunol., 11: 98 - 107. DOI: 10.1038/nri2925

FaraG, Y. M., Gaballa, M. R. (2011): Diabesity: an overview of a rising epidemic. Nephrol. Dial. Transplant., 26: 28 - 35. DOI: 10.1093/ndt/gfq576

Freigang, S., Ampenberger, F., Weiss, A., Kanneganti, T. D., Imakura, Y., Hersberger, M., Kopf, M. (2013): Fatty acid-induced mitochondrial uncoupling elicits inflammasome-independent IL-1alpha and sterile vascular inflammation in atherosclerosis. Nat. Immunol., 14: 1045 - 1053.10.1038/ni.2704

JIN, M. S., LeE, J. O. (2008): Structures of the toll-like receptor family and its ligand complexes. Immunity, 29: 182 - 191. DOI: 10.1016/j.immuni.2008.07.007

Jourdan, T., Godlewski, G., Cinar, R., Bertola, A., Szanda, G., Liu, J., Tarn, J., Han, T., Mukhopadhyay, B., Skarulis, M. C., Ju, C., Aouadis, M., Czech, M., Kunos, G. P. (2013): Activation of the Nlrp3 inflammasome in infiltrating macrophages by endocannabinoids mediates beta cell loss in type 2 diabetes. Nat. Med., 19: 1132 1140. DOI: $10.1038 / \mathrm{nm} .3265$

KaJl, N., Okuno, A., Ohno-Ichikı, K., OKI, H., Ishizawa, H., ShIRAl, M., AsAl, F. (2012): Plasma profiles of glucose, insulin and lipids in the male WBN/Kob-Lepr(fa) rat, a new model of type 2 diabetes with obesity. J. Vet. Med. Sci., 74: 1185 - 1189. DOI: 10.1292/ jvms.12-0045

Kamari, Y., Shaish, A., Shemesh, S., Vax, E., Grosskopf, I., Dotan, S., White, M., Voronov, E., Dinarello, C. A., Apte, R. N., Harats, D. (2011): Reduced atherosclerosis and inflammatory cytokines in apolipoprotein-E-deficient mice lacking bone marrow-derived interleukin-1alpha. Biochem. Biophys. Res. Commun., 405: 197 203. DOI: 10.1016/j.bbrc.2011.01.008

Kamari, Y., Werman-Venkert, R., Shaish, A., Werman, A., Harari, A., Gonen, A., Voronov, E., Grosskopf, I., Sharabi, Y., Grossman, E., Inakura, Y., Dinarello, C.A., Apte, R.N., Harats, D. (2007): Differential role and tissue specificity of interleukin-1alpha gene expression in atherogenesis and lipid metabolism. Atherosclerosis, 195: 31 - 38.10.1016/j.atherosclerosis.2006.11.026

Kawal, T., AkIRA, S. (2011): Toll-like receptors and their crosstalk with other innate receptors in infection and immunity. Immunity, 34: 637 - 650. DOI: 10.1016/j.immuni.2011.05.006

Maedler, K., Sergeev, P., Ris, F., Oberholzer, J., Joller-Jemelka, H. I., Spinas, G. A., Kaiser, N., Halban, P. A., Donath, M. Y. (2002): Glucose-induced beta cell production of IL-1beta contributes to glucotoxicity in human pancreatic islets. J. Clin. Invest., 110: 851 860. DOI: $10.1172 /$ jci15318

Nagakubo, D., Shiral, M., Nakamura, Y., Kaul, N., Arisato, C., Watanabe, S., Takasugi, A., Asal, F. (2014): Prophylactic effects of the glucagon-like Peptide-1 analog liraglutide on hyperglycemia in a rat model of type 2 diabetes mellitus associated with chronic pancreatitis and obesity. Comp. Med., 64: 121 - 127

Oкамото, M., TalRa, K., Iто, R., Asal, F. (2015): Transcriptomic study of the rat pinworm Syphacia muris. Helminthologia, 52: 370 - 374. DOI: 10.1515/helmin-2015-0059

Okamoto, M., Van Stry, M., Chung, L., Koyanagi, M., Sun, X., SuZuKI, Y., Ohara, O., Kitamura, H., HiJikata, A., Kubo, M., Bix, M. (2009): Mina, an II4 repressor, controls T helper type 2 bias. Nat. Immunol., 10: 872 - 879. DOI: 10.1038/ni.1747

Okuno, A., KaJl, N., Takahashi, A., Nagakubo, D., Ohno-lchikı, K., SHIRAI, M., AsAl, F. (2013): Role of insulin resistance in the pathogenesis and development of type 2 diabetes in WBN/Kob-Lepr(fa) rats. J. Vet. Med. Sci., 75: 1557 - 1561. DOI: 10.1292/jvms.130230

Rider, P., Carmi, Y., Voronov, E., Apte, R. N. (2013): Interleukin-1alpha. Semin. Immunol., 25: 430 - 438. DOI: 10.1016/j. smim.2013.10.005

Rodriguez-Hernandez, H., Simental-Mendia, L. E., Rodrlguez-Ramirez, G., Reyes-Romero, M. A. (2013): Obesity and inflammation: epidemiology, risk factors, and markers of inflammation. Int. J. Endocrinol., 2013: 678159. DOI: 10.1155/2013/678159 
Sorrentino, R., Terlizzi, M., Di Crescenzo, V. G., Popolo, A., Pecoraro, M., Perillo, G., Galderisi, A., Pinto, A. (2015): Human lung cancer-derived immunosuppressive plasmacytoid dendritic cells release IL-1alpha in an AIM2 inflammasome-dependent manner. Am. J. Pathol., 185: 3115 - 3124. DOI: 10.1016/j.ajpath.2015.07.009

Spears, L. D., Razani, B., Semenkovich, C. F. (2013): Interleukins and atherosclerosis: a dysfunctional family grows. Cell Metab., 18: 614 - 616. DOI: 10.1016/j.cmet.2013.10.009

Staht, W. (1961): Syphacia muris, the Rat Pinworm. Science (New York, N.Y.), 133:576 - 577. DOI: 10.1126/science.133.3452.576-a Taira, K., Yazawa R., Watanabe, A., Ishikawa, Y., Оkamoto, M., TakaHASHI, A., AsAl, F. (2015): Syphacia muris infection delays the onset of hyperglycemia in WBN/Kob-Leprfa rats, a new type 2 diabetes mellitus model. Helminthologia, 52: 58 - 62. DOI: 10.1515/ helmin-2015-0010

Wen H., Gris D., Lei Y., Jha S., Zhang L., Huang M. T., Brickey W. J., TING, J. P. (2011): Fatty acid-induced NLRP3-ASC inflammasome activation interferes with insulin signaling. Nat. Immunol., 12: 408 - 415. DOI: 10.1038/ni.2022

Zimmet, P., AlbertI, K.G., Shaw, J. (2001): Global and societal implications of the diabetes epidemic. Nature, 414: 782 - 787. DOI: 10.1038/414782a

ZUCKER, L.M. (1965): Hereditary obesity in the rat associated with hyperlipemia. Ann. N. Y. Acad. Sci., 131: 447 - 458. DOI: 10.1111/ j.1749-6632.1965.tb34810.x 